\title{
Urgences
}

\section{La littérature enfantine à l'école}

\section{Pierrette Santerre et Ginette Tremblay}

Numéro 8, 4e trimestre 1983

Littérature jeunesse

URI : https://id.erudit.org/iderudit/025114ar

DOI : https://doi.org/10.7202/025114ar

Aller au sommaire du numéro

Éditeur(s)

Urgences

ISSN

0226-9554 (imprimé)

1927-3924 (numérique)

Découvrir la revue

Citer cet article

Santerre, P. \& Tremblay, G. (1983). La littérature enfantine à l'école. Urgences, (8), 7-18. https://doi.org/10.7202/025114ar d'utilisation que vous pouvez consulter en ligne.

https://apropos.erudit.org/fr/usagers/politique-dutilisation/ 


\section{CHRONIQUE}

\section{LA LITTÉRATURE ENFANTINE}

À L'ÉCOLE

\section{par}

Pierrette Santerre et Ginette Tremblay enseignantes, C.S. La Neigette 


\section{LA LITTÉRATURE ENFANTINE À L'ÉCOLE \\ PLACE AUX LIVRES}

Un des rôles qu'on a toujours donné à l'école est celui d'apprendre à lire à l'enfant et cela, dès la première année du Primaire, si possible. L'enfant sait très bien lire dans son manuel de lecture mais est-ce suffisant pour lui donner le goût de lire? L'école doit donc dépasser son rôle d'enseignement et développer chez I'enfant des habitudes de lecteur créateur. Le goût de lire n'est pas inné, il naît dans un milieu où la lecture a une raison d'être.

Souvent on prive l'enfant de livres parce que le livre de jeunesse nous est peu familier. On s'interroge sur la façon d'amener le livre à l'école, comment l'intégrer à la vie de la classe et comment amener l'enfant à vivre ses lectures. On manque de sécurité devant une nouvelle approche du livre, on hésite à prendre des risques, à expérimenter de nouvelles données pédagogiques; on manque d'aide, de temps et surtout d'information. On veut bien faire quelque chose pour intégrer la littérature enfantine - sans pour autant scolariser la lecture - mais par quoi commencer? Peu importe les moyens qu'on décidera de prendre, une condition est essentielle, c'est d'abord d'AIMER LA LITTEERATURE ENFANTINE. Quand on aime quelque chose, c'est facile d'en parler!

Depuis quelques années, nous avons vécu quelques expériences dans le but de faire vivre le livre dans la classe. Nous présenterons quelques stratégies permettant de promouvoir la littérature enfantine à l'école. Nous insisterons d'abord sur une formule qui permet à l'enfant d'amener le livre en classe - l'aire de lecture et nous poursuivrons avec quelques projets gravitant autour du livre. En plus de faire connaître les livres, ces activités permettent à l'enseignante de s'informer des goûts, des intérêts et des habiletés de lecture des enfants. 


\section{CONNAÎTRE LA LITTÉRATURE ENFANTINE}

\section{AIRE DE LECTURE}

Pour que les enfants lisent, il faut d'abord que les enfants soient en contact direct avec les livres. Les livres doivent faire partie de l'environnement de la classe; ils doivent être présents, vivants, à la portée des enfants. Ce n'est pas une heure hebdomadaire figée et fixée à l'horaire qui va donner le goût de lire à l'enfant. Malheureusement, très souvent, le fonctionnement de la bibliothèque scolaire ne permet pas I'accessibilité aux livres en tout temps. A court terme, une solution à envisager est sûrement d'amener le livre dans la classe en y installant une aire de lecture. Mais avant d'aménager un espace pour la lecture', l'enseignante doit d'abord susciter un intérêt chez les enfants en leur faisant désirer ce coin et en les impliquant personnellement dans cette organisation. Un coin de lecture désiré et organisé par des mains d'enfant est sûrement plus efficace qu'un coin préparé par l'enseignante seule. L'organisation matérielle comme le choix de l'espace, la décoration, la disposition, le choix du matériel, les règles de vie et la gestion, doivent revenir aux enfants. II va de soi que tout au long de ce projet, l'enseignante devra soutenir l'intérêt et guider les enfants.

Une aire de lecture, même si elle est bien organisée, doit être animée constamment par l'enseignente. C'est dans la mesure où l'enseignante s'intéresse à cet espace réservé à la lecture et lui accorde une grande importance que l'enfant aimera le fréquenter. Pour maintenir cet intérêt, l'enseignante peut imaginer plusieurs stratégies:

- renouveler régulièrement les livres en invi- 
tant les écoliers à apporter leurs livres de la maison. $C^{\prime}$ est une excellente occasion de connaître ce que les enfants lisent et de s'informer de leurs goûts en lecture. C'est aussi le bon moment pour l'enfant de présenter son livre préféré La chicane, inviter ses amis à lire Émilie, la baignoire à pattes ou encore faire connaître son héros favori de B.D. (bandes dessinées);

- éveiller l'attention aux nouveautés. Un lutrin peut très bien servir pour présenter le "livre vedette" de la semaine. Un ami peut aussi y exposer un livre qu'il aime bien: Tourbillon, le lutin de la Côte Nord;

- inviter les écoliers à afficher leurs poèmes, leurs histoires, leurs réalisations et à promouvoir euxmêmes la littérature à l'aide de pancartes, de messages, d'affiches, etc... ("Tu veux bien rigoler? Tu ne te tromperas pas en lisant Alexis le trotteur".);

- changer fréquemment la présentation des livres par de nouvelles classifications inventées par les enfants. (Les enfants classent les livres par grandeur, par collection, par genre, etc...).

Pour le succès de l'aire de lecture, I'enfant doit d'abord se sentir à l'aise, y avoir accès librement, avoir le choix de ses lectures, être impliqué dans son fonctionnement et surtout avoir du temps pour lire.

L'aire de lecture - souvent appelé coin de lecture - est une bonne stratégie pour amener l'enfant à lire mais il ne doit absolument pas remplacer la bibliothèque. Bibliothèque et aire de lecture doivent se compléter, s'enrichir réciproquement, être intégrées au vécu de la classe et de l'école et offrir un éventail de livres variés. 


\section{CHOIX DE LIVRES}

Comment choisir les livres qui alimentent nos bibliothèques et nos aires de lecture quand on connaît à peine les livres? II est d'abord important de s'associer les enfants pour un meilleur choix possible. En observant les enfants lire, manipuler les livres, échanger sur leurs lectures, discuter, on apprend vite à connaître leurs goûts, leurs intérêts et leurs habiletés à lire. C'est en vivant des projets variés que nous nous sommes familiarisées avec la littérature enfantine et que nous avons appris à connaître les lecteurs. Le's quelques expériences vécues ont été tentées avec les enfants en collaboration avec des parents, des collègues de l'école, des libraires, des bibliothécaires et quelques auteurs. Ces projets se résument à des activités collectives:

- une nouvelle classification des livres de la bibliothèque de l'école où les enfants "brassent" les livres, les manipulent, les rangent d'une façon pratique. Ils ont la chance et surtout le temps de fouiner, de regarder ce qu'il y a à lire. On démystifie ce lieu de silence pour en faire un endroit où on a le goût d'aller lire, d'échanger ses lectures, de s'exprimer et de partager.

- I'organisation d'un mini-salon du livre dans la classe où les bureaux servent de présentoirs; où les coins de bicolage, de bric-à-brac, l'aquarium et le castelet sont prétextes aux livres; où parents et amis de l'école sont invités à visiter.

- des visites régulières à la bibliothèque publique où des nouveaux membres s'inscrivent et d'autres échangent leurs livres. C'est une façon pour les enfants de lire à bon compte! 
- des visites aux librairies locales où les jeunes lecteurs découvrent des "magasins de livres" et feuillettent les nouveautés.

- et des rencontres avec des auteurs où les enfants apprennent à connaître le métier d'écrivain. Rencontrer un auteur ${ }^{2}$ pour les enfants, c'est un peu un rêve devenu réalité. Les enfants réalisent que l'auteur est quelqu'un qui écrit pour eux dans le but de les divertir, de les convaincre, de les informer ou de les faire agir. Cette rencontre suscite chez les jeunes lecteurs un certain intérêt pour les livres de l'auteur et permet ainsi un véritable échange.

Les réactions des enfants et les échanges constants avec ceux-ci au cours de ces activités nous ont révélé que les enfants d'un même groupe d'âge ont des goûts très variés, des intérêts différents et qu'ils sont attirés par les thèmes ou les sujets les plus divers. Ce sont des observations à considérer dans un choix de livres.

De plus, il est fort avantageux de consulter des outils d'information ${ }^{3}$. Ils nous font connaître les nouveautés, nous fournissent des critiques intéressantes de livres et nous facilitent ainsi le choix d'une variété de livres. 


\section{PROMOUVOIR LA LITTÉRATURE ENFANTINE}

\section{LE LIVRE EN ÉVIDENCE DANS LA CLASSE}

La présence de livres variés à l'école ne suffit pas à maintenir l'intérêt à la lecture. Ce n'est pas tout de mettre les livres dans les mains des enfants et les laisser lire, encore faut-il faire vivre le livre dans la classe. Bien qu'un espace spécifique réservé à la lecture l'aire de lecture - motive l'enfant à lire, il ne doit pas être le seul lieu privilégié du livre. Pour maintenir l'intérêt des enfants aux livres, on doit "déborder" de l'aire de lecture et faire de la classe l'endroit idéal pour la promotion du livre. À l'occasion, on étale de la documentation sur une table; des nouveautés littéraires sur le rebord du tableau; on suspend les revues Hibou à une ficelle; on étale la collection Pour lire avec toi sur le rebord des fenêtres; on dépose différents dictionnaires dans un bac; on place les Petit As au coin de bricolage, etc... II est nécessaire de faire circuler les livres de la maison à l'école, de la bibliothèque à la classe et de la classe à la maison, de présenter les livres et de les faire désirer.

\section{STRATÉGIES DE PROMOTION DU LIVRE}

L'enseignante peut imaginer de nombreuses stratégies pour présenter les livres tout en tenant compte de son groupe d'écoliers, des réalités de la classe et des conditions matérielles de son école. Ces stratégies peuvent être de simples activités autour du livre ou prendre la forme de projets. Pour que les activités de promotion du livre soient intégrées à la vie de la classe, il est nécessaire que l'enseignante ait une bonne connaissance des livres et qu'elle planifie son programme d'actions. Par exemple, elle retient $\mathrm{La}$ famille $\mathrm{Ci}$ - 
trouillard au temps des sucres pour préparer une visite à la cabane; elle présente Pitatou et les pommiers au temps des pommes; elle suggère Petite fleur et Brin $d^{\prime}$ herbe lors d'une randonnée au parc. Elle doit aussi être à l'écoute des enfants et être habile à saisir les situations spontanées où c'est le bon moment de présenter le bon livre. Elle organise une semaine de la poésie où elle fait rythmer par les petits les comptines Des comptines traditionnelles du Canada français. Elle leur fait fredonner les chansons de Grand-père Cailloux, tirées de Mon petit lutin s'endort et Je te laisse une caresse et les invite à mémoriser "Gros minou" dans Un clin d'oeil de Grand-maman aux coccinelles. Elle éveille les plus grands à l'expression poétique en lisant Gens de mon pays et Un clin d'oeil de Grandmaman aux hirondelles. Elle choisit un moment précis appelé "L'heure du Conte" pour faire la lecture d'Étoilefilan, Les perles de pluie, Les quatre saisons de Piquot. Elle fait observer les illustrations de La surprise de dame chenille et invite les tout-petits à lire l'image dans La Cachette.

La plupart du temps, ces activités de promotion du livre se vivent dans un climat de détente où les enfants sont regroupés autour de l'enseignante. Souvent, elle amorce ces activités par une approche oralisée - sous forme de dialogue - et crée ainsi une véritable situation de communication.

À lire avec les enfants, on apprend à aimer les livres et à découvrir les multiples qualités des livres. Elles sont des sources possibles d'exploitation pour amener les enfants à faire de vraies lectures. L'exploitation du livre est une autre étape à entreprendre pour développer chez l'enfant des habitudes de lecteur créateur. 


\section{LISTE DES TITRES CITÉS DANS CET ARTICLE DANS L'ORDRE DE PRÉSENTATION}

La chicane. Ginette Anfousse, ill. I'auteur, éd. La courte échelle, coll. L'étoile filante, 1976, $24 \mathrm{p}$.

Émilie, la baignoire à pattes. Bernadette Renault, ill. Félix Vincent, éd. Héritage, coll. Albums Héritage, 1978, 16 p.

Tourbillon, le lutin de la Côte Nord. Francine Loranger, ill. Danielle Shelton, éd. Héritage, coll. Pour lire avec toi, 1977, 121 p.

Alexis le trotteur, I'homme qui courait comme un cheval. Blaise, ill. Bos., éd. Paulines, coll. Loisirs VIP, Montréal, 1979, $38 \mathrm{p}$.

Hibou, le nouveau magazine des jeunes. éd. Héritage inc., Montréal.

La collection "Pour lire avec toi", éd. Héritage, Montréal.

La collection "Petit As", éd. École Active/Gamma, 1975.

La famille Citrouillard au temps des sucres. Rita Scalabrini, ili. de l'auteur, éd. Leméac, 1976. 32 p.

Pitatou et les pommiers. Louise Pomminville, ill. par l'auteur, éd. Leméac, coll. "Les merveilleux oiseaux de la forêt de nulle part", 1972.

Petite fleur. Le groupe Fleurbec, auteur et éditeur, distribution Fides, 1981. $12 \mathrm{p}$.

Brin d'herbe. Le groupe Fleurbec, auteur et éditeur, distribution Fides, 1981, $12 \mathrm{p}$.

Comptines traditionnelles du Canada français. Dessins de Michèle Leclerc, Louise Méthé, Yolande Chatillon, éd. Leméac, coll. Littérature de jeunesse, 1973. 32 p. 
Mon petit lutin s'endort. Grand-père Cailloux, ill. Gilles Tibo, éd. Le Tamanoir, coll. L'étoile filante, Montréal, 1976. 24 p.

Je te laisse une caresse. Grand-père Cailloux, ill. Gilles Tibo, éd. La courte échelle, coll. L'étoile filante, 1976, 24 p.

Un clin d'oeil de Grand-maman aux coccinelles. Corinne Normand-Hudon, ill. Suzanne Michaud, éd. Hurtubise, $\mathrm{HMH}$, 1977. 74 p.

Les gens de mon pays. Gilles Vigneault, ill. Miyuki Tanobe, éd. La courte échelle, Montréal, 1980, 24 p.

Un clin d'oeil de Grand-maman aux hirondelles. Corinne Normand-Hudon, ill. Suzane Michaud, éd. Hurtubise HMH, 1977. $77 \mathrm{p}$.

Etoifilan. Bertrand Gauthier, ill. Gilles Pednault, éd. Le Tamanoir, coll. L'étoile filante, 1975. 32 p.

Les perles de pluie. Eric Mérinat, ill. Lucienne Fontannaz, éd. La courte échelle, 1979, 24 p.

Les quatre saisons de Piquot. Gilles Vignault, ill. Hugh John Barrett. Livre-disque. Nouvelles éditions de I'arc, 1979. 36 p.

La surprise de dame chenille. Henriette Major, ill. Claude Lafortune, éd. CPP, dist. Leméac, coll. Premier pas, 1970.48 p.

La cachette. Ginette Anfousse, ill. de l'auteur, éd. La courte échelle, coll. Etoile filante, Montréal, 1976. 24 p.

\section{NOTES}

(1) Pour faciliter l'aménagement et I'animation d'un coin de lecture, vous pouvez consulter:

Pédagogie et lecture

Denise Bourneuf et André Pari,

Ed. Québec/Amérique, 1975. 
(2) C'est assez facile d'organiser une rencontre avec un auteur. II existe un organisme sans but lucratif, Communication-jeunesse, qui fait la promotion de la littérature de jeunesse depuis plus de dix ans et facilite ces rencontres auteurs-lecteurs. Pour tout renseignement concernant ces rencontres:

Communication-jeunesse

445, rue Saint-François-Xavier

Montréal, Québec

H2Y 2T1 Tél.: 844-5939

(3) Pour mieux connaître et choisir le livre de jeunesse, nous vous suggérons quelques outils d'information: - Choix Jeunesse, documentation imprimée (4 fois I'an)

Choix-Jeunesse

Centrale des bibliothèques 1685 , rue Fleury est

Montréal, Québec H2C 1T1

- Des livres et des jeunes, revue pour l'avancement de la littérature de jeunesse

Des livres et des jeunes/ACALJ

C.P. 2152, succursale Jacques-Cartier

Sherbrooke, Québec J1K 9C9

- Lurelu, le trimestriel québécois de la littérature de jeunesse ( 3 numéros par an)

Lurelu

Case postale 446

Succ. Delorimier

Montréal, Québec H2N 1N7 
- Fascicule 2. Bibliographie sélective commentée de livres québécois,

Ministère de l'Éducation, 1981.

No. 16-2410-04 par Francine C. Lebel

et Michelle Provost

- Mise à jour du fascicule 2. Littérature de jeunesse québécoise 1979-1980. Une sélection de livres de fiction.

Vie pédagogique

Éditeur officiel du Québec

No. 15, nov. 1981 (p. 36-45)

- Mise à jour du fascicule 2. Des livres québécois pour les jeunes: 1981, un bon cru.

Vie pédagogique

Éditeur officiel du Québec

No. 18, avril 1982 (p. 21-29)

- Fascicule 3. Bibliographie sélective commentée de livres francophones publiés à l'étranger,

Ministère de l'Éducation, 1981.

par Michelle Provost et Jani Pascal,

no. $16-2410-05$

- Vie pédagogique,

Ministère de l'Éducation

Direction des communications

1035 De la Chevrotière

Québec G1R 5A5

- La revue des livres pour enfants, Paris, France (6 numéros par an)

La joie par les livres

4 , rue de Louvois

75002 Paris, France 